\title{
V027 THE INTEGRATED APPROACH TO RESERVOIR EVALUATION - MYTHS AND REALITIES
}

ELIO POGGIAGLIOLMI

EnTec Energy Consultants Ltd, Melrose House, 42 Dingwall Road, Croydon Surrey CRO 9NE, UK

There is general consensus among oil and service companies that total integration of all available data by a multi-disciplinary team is a more cost effective and productive method of reservoir evaluation than the conventional isolated piecemeal approach. To this end, multi-disciplinary teams formed to work on specific fields are a common occurrence. These teams are composed of individuals that not only belong to different disciplines but also to different cultures.

"Brain storming" sessions are often considered the most effective means to promote communication, by breaking down culture barriers and encouraging interdisciplinary cross-fertilization. Unfortunately, brain storming can be at best a palliative because most of the effort by team members is dedicated to carrying out the tasks related to their specific area of expertise. For example, the geophysicist deals with the various aspects of seismic data, the log analyst with borehole data, etc. These tasks are normally carried out independently and with a minimum of communication and interaction among the team members. Often the segregation of tasks is attributed to the lack of integrated software and insufficient knowledge of disciplines outside the individual's area of expertise. A typical example, resulting from this segregation of tasks is the use of 3D surveys. Normally, these are used to obtain accurate geometric definition of the reservoir - a task with which the geophysicist feels very much at home! However, the full potential of 3D to obtain a quantitative knowledge of the porosity, fluids and lithological distribution is very much neglected, since this will involve not only seismic, but also the contribution from log analysis, petrophysicists, geologists, and engineers. Such contribution must not be a collection of piecemeal results by team members, e.g. structure maps, geological models, formation evaluation results, etc. For integration to be meaningful, it must start from raw measurements with each member of the team working together throughout all stages of the data processing. Total interaction and feedback must take place through integrated software and effective human communication. The work areas must be shared among the team members without physical barriers. This encourages human communication and exchange of ideas. Unless this approach is adopted, the available data is under-utilized, thereby reducing the cost-effectiveness and productivity.

Another obstacle on the path to integration is budgetary segregation. Budgets are assigned to carry out individual tasks within exploration, development, or production. Very seldom are integrated budgets available to carry out simultaneously a multitude of tasks. This is one of the main reasons why there is lack of motivation on the part of the service industry to invest resources for, a more integrated approach.

For the integration to be useful and cost effective, it must meet the following criteria:

- assign field specific integrated budgets

- multi-disciplinary training of team members

- provide software designed "to force" each user to utilize data and results from other users

- start integration from raw measurements

- provide common work areas for the team

In conclusion, total integration can bring the following benefits when properly carried out:

- full and efficient use of available information

- more accurate and reliable results

- cost effectiveness and added value

- effective use of all available resources

- systematic solution to the problem in hand

- possibility of solving otherwise intractable and/or non-unique problems

- better bottom line budget control

Case history examples will be given to illustrate the results obtained by adopting a fully integrated approach to reservoir evaluation. 
